\title{
Spanish Initiative for the Automation in Urban Transport: AutoMOST
} \author{
Ray A. Lattarulo ${ }^{1}$ \\ ${ }^{1}$ Industry \& Transport Division, Tecnalia, Derio, Spain \\ ${ }^{2}$ Grupo Avanza, Madrid, Spain \\ Email: jesus.murgoitio@tecnalia.com
}

Jesús Murgoitio', Rafael Durbán², Joshué M. Pérez¹, Antonio García², Jose A. Moreno²,

How to cite this paper: Murgoitio, J., Durbán, R., Pérez, J.M., García, A., Moreno, J.A. and Lattarulo, R.A. (2018) Spanish Initiative for the Automation in Urban Transport: AutoMOST. Journal of Transportation Technologies, 7, 1-10. https://doi.org/10.4236/jtts.2018.81001

Received: September 11, 2017

Accepted: November 5, 2017

Published: November 8, 2017

Copyright $(9) 2018$ by authors and Scientific Research Publishing Inc. This work is licensed under the Creative Commons Attribution International License (CC BY 4.0).

http://creativecommons.org/licenses/by/4.0/

\begin{abstract}
The progressive automation of transport will imply a new paradigm in mobility, which will profoundly affect people, logistics of goods, as well as other sectors dependent on transport. It is precise within this automation where the development of new driving technologies is going to cause a great impact on the mobility of the near future, and that will have an effect on the economic, natural and social environment. It is therefore a primary issue at the global level, as it is reflected in the work programs of the European Commission in relation to the road transport [1] [2]. Thus, the size impact is caused by the following novelties and advantages: 1) Safety: Accidents reduction caused by human error; 2) Efficiency increase in transportation, both in energy consumption and time; 3) Comfort for users and professionals who will increase their operational availability to execute other more valuable tasks, both for them and enterprises; 4) Social Inclusion: enabling mobility easily for everybody during more time; 5) Accessibility, to get to city centers and other difficult reach places. It should be noted that the economic impact projected for automated driving for the years to come ranges up to $€ 71$ bn in 2030, when estimated global market for automated vehicles is 44 million vehicles, as is reflected in document Automated Driving Roadmap by ERTRAC [3], European Road Transport Research Advisory Council

(http://www.ertrac.org/uploads/documentsearch/id38/ERTRAC_AutomatedDriving-2015.pdf). As background that already anticipates these improvements, the Advance Driver Assistance System (ADAs) have already showed the safety increase in the last ten years, but always maintain a leading role for the driver. Related to the efficiency increase, automated driving offers great opportunities for those companies where mobility is a key factor in operating costs, and affects the whole value chain. The project opportunity is consistent
\end{abstract}


with ERTRAC vision, especially in applications focused on the urban environment [4], where it is expected a deployment of the technology of high level automation in an immediate future. This is possible by the potential to incorporate smart infrastructure to improve guidance and positioning, as well as lower speed, which eases its progressive deployment. The objective of AutoMOST is developing technologies for the automation of vehicles in urban transport and industrial applications, to increase significantly the efficiency, safety and environmental sustainability. More specifically, AutoMOST will allow the implementation of shared control systems (Dual-Mode) [5] for future automated vehicles that allow the services operate more efficiently and flexibly, in a context of intelligent and connected infrastructures.

\section{Keywords}

Urban Transport, Automated Vehicles, Dual Mode

\section{Introduction}

Transport automation will significantly affect people's lives, merchandise transport and other transport-related sectors [6] [7]. It is precise in this context of progressive automation that the development of new technologies applied to transport becomes an aspect vital to the competitiveness of companies engaged in mobility, affecting their future positions in the economic, environmental and social areas. For all these reasons, road transport automation currently represents a global priority concern, reflected in the work programs of the European Commission [1] [2] concerned with research into land transport. In more concise terms, the benefits expected from transport automation are described below:

- Safety: The reduction of accidents associated to human error.

- Increased transport efficiency (energy consumption, etc.).

- Comfort for users and professionals, increasing resource availability for other tasks.

- Social inclusion, making mobility for everybody possible for longer periods.

- Accessibility in reaching city centres and other areas of difficult access.

With regard to increased efficiency, vehicle automation offers great opportunities for those companies, to whom mobility is critical to operating costs, affecting the entire value chain [7]. In this sense, not only private cars will benefit from the advantages of automation; passenger transport companies, logistics, and other sectors of the urban setting will also benefit from the boon that automated driving offers.

Specifically, AutoMOST, which main information is shown in Figure 1 (see more detailed Figure 2 \& Figure 3 too), will enable the implementation of shared (dual mode) control systems for future automated vehicles that will allow for the more efficient and flexible operation of services in a context of interconnected smart infrastructures. 


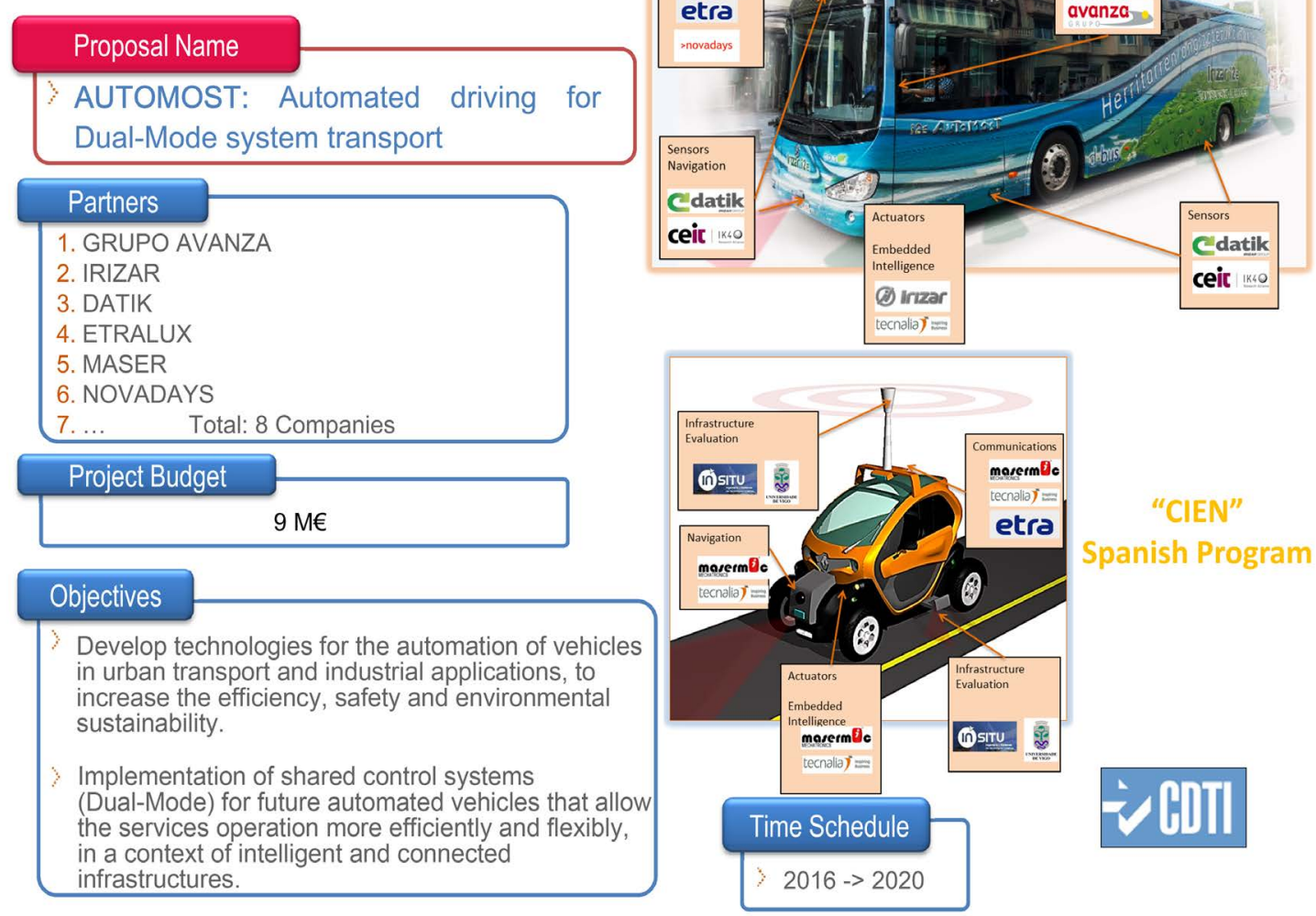

Figure 1. Summarized project data.

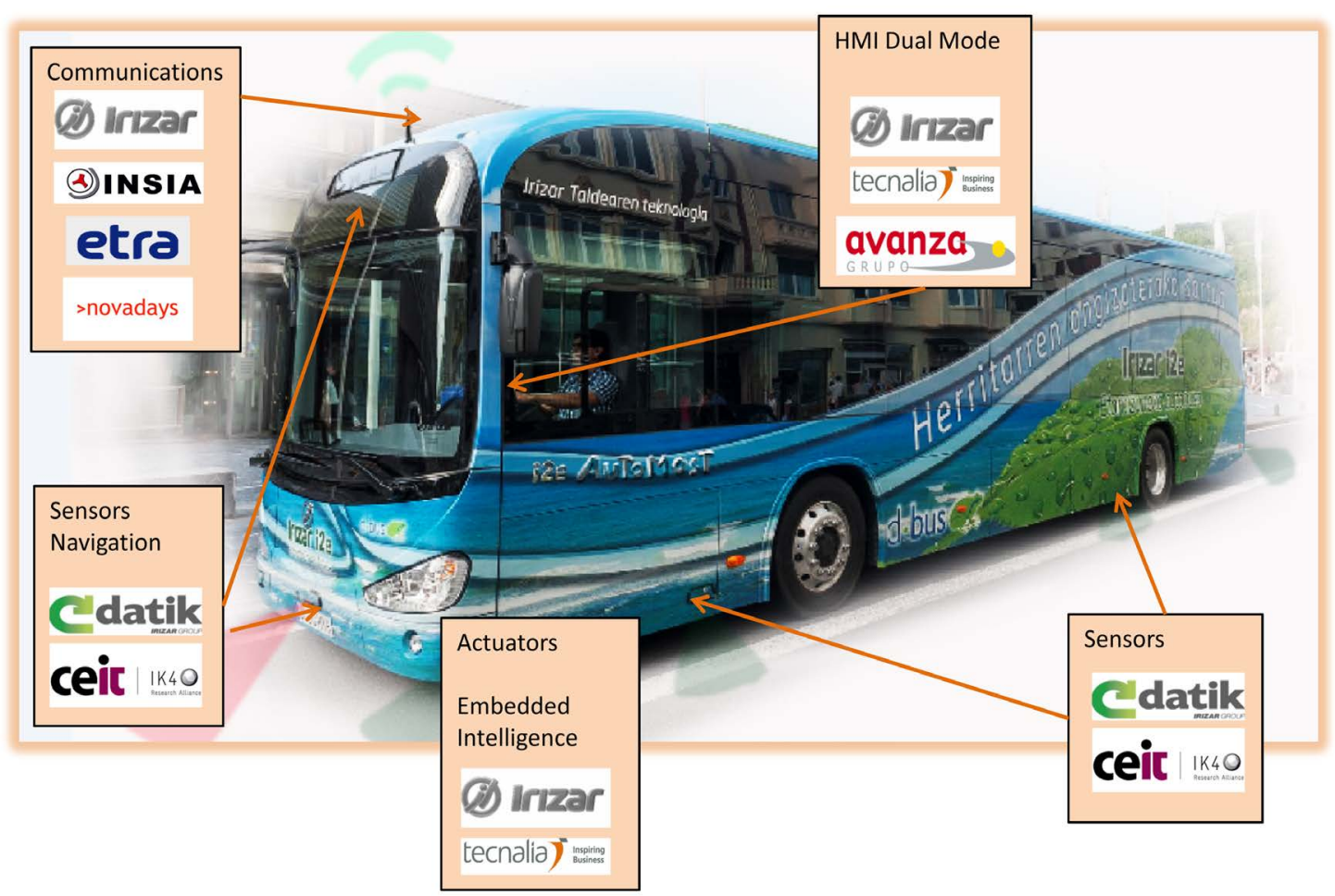

Figure 2. Partner contributions in use case 1: electric bus. 


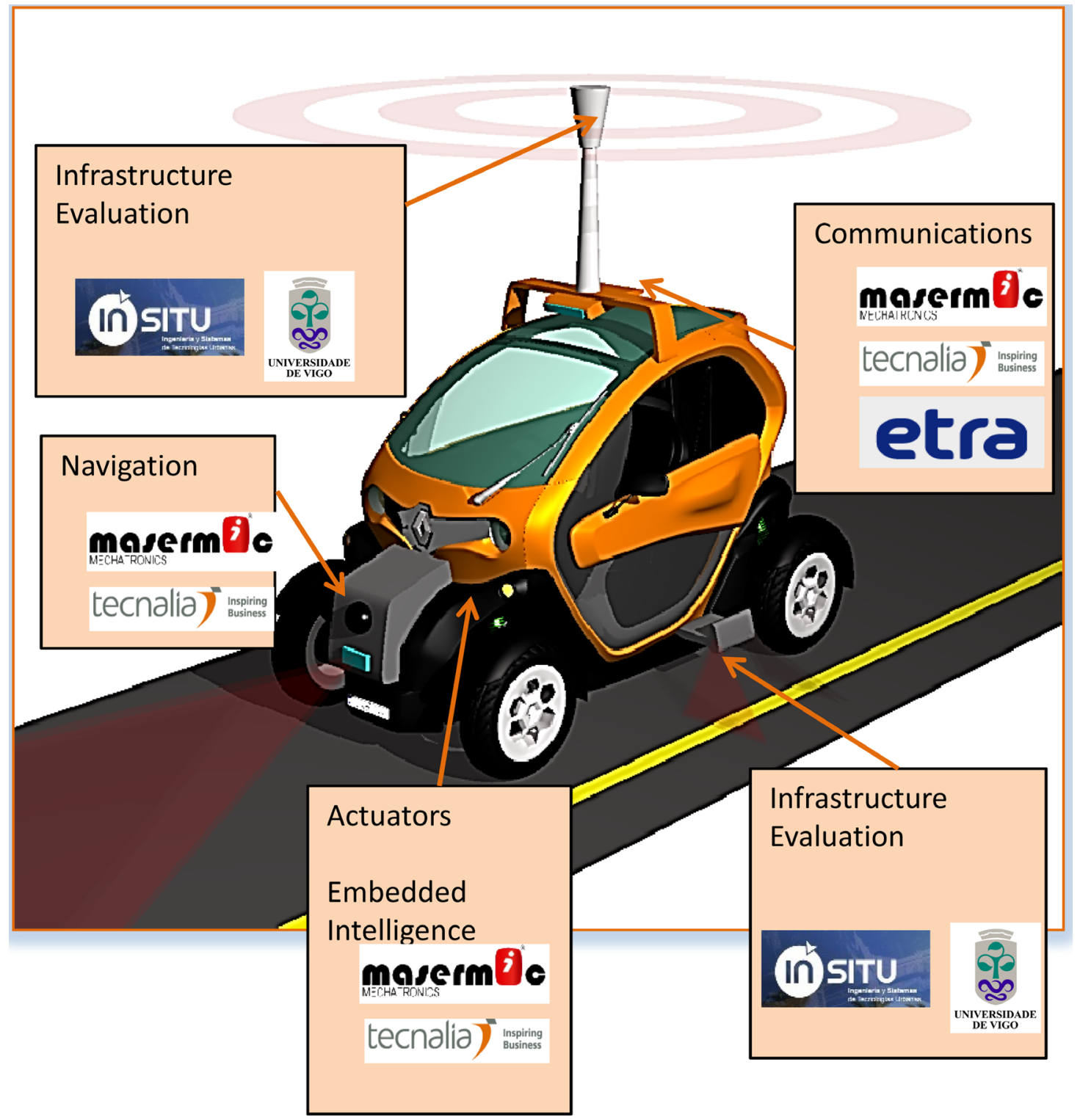

Figure 3. Partner contributions in use case 2: infrastructure maintenance vehicle.

AutoMOST approaches the research and development of enabling elements holistically, simultaneously affecting the value chain to ensure high industrial impact and global leadership positioning for the companies comprising the consortium. In doing this, it departs from the application of existing functions, such as the following driving assistance systems [8]: Adaptive Cruise Control (ACC), Lane Keeping System (LKS), pedestrian detection, smart infrastructures... to facilitate highly efficient operating systems for companies and infrastructures providing mobility, while demonstrating operational cost reductions in specific applications.

Figure 4 shows the advantage of project opportunity, according to a recent study by Morgan Stanley (Company Data, Morgan Stanley Research), which is consistent with ERTRAC vision, especially in applications focused on the urban environment [4]. 


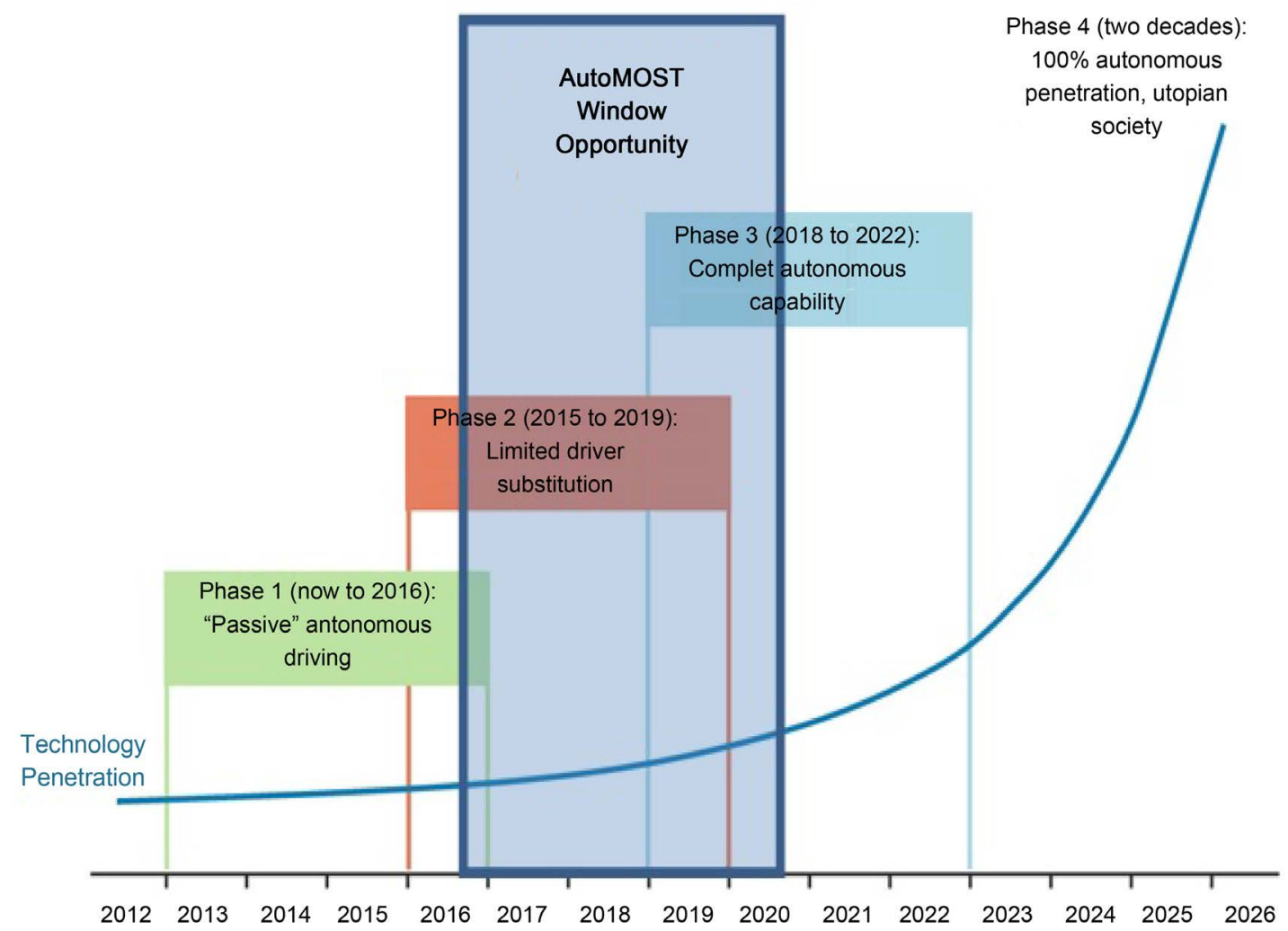

Figure 4. Timeline for technology adoption. Source: Company data, Morgan Stanley research.

\section{Approach}

The general purpose of AutoMOST is to develop technologies enabling vehicle automation for application to urban and industrial transport with a view to significantly increasing efficiency, safety and sustainability [9].

\subsection{Scientific and Technological Objectives}

Following are the scientific and technological project objectives arising from the aforesaid general purpose.

- To design and implement a control architecture for automated vehicles of different types (passenger car/large bus), defining each of the blocks in this architecture in a modular way so as to be easily interchangeable, and implementing the different advances in keeping with the new electric/electronic vehicle architectures (in line with standards).

- To implement on-board perception systems for automated vehicles in urban and inter-urban settings, as well as to design controllers for steering (lateral driving) and for pedals (longitudinal driving) that can be used individually and jointly.

- To implement a global and local route generator for real-time vehicle path definition and optimum velocity profile definition based on the data supplied by 3D maps, communication systems, vehicle models and propulsion system status. 
- To implement a communications system between vehicles and infrastructures (V2I) that will enable optimizing previously defined profiles and routes, along with the development of infrastructure systems (control centre) for operating the system safely, in tandem with traffic management.

\subsection{Technical Approach and Structure of Activities}

Over the last few years, automated vehicles have been accepted by a great proportion of car manufacturers [7]. The benefits this can bring to transport, along with infrastructure, social, safety [9], emission and other advantages, place us squarely on the path to one of the ground-breaking technologies of the 21 st century.

\subsubsection{Technical Approach}

AutoMOST focuses on the use of many of the functions already existing for automated vehicles up to the present, avoiding additional efforts with regard to systems already tested and validated. AutoMOST seeks to go beyond by developing technologies that enable automated "dual mode" driving under real traffic conditions, interacting safely with the dynamic urban environment and with expert drivers to validate interaction between different automation levels [3] and thus allow for safe, efficient deployment.

The control architecture to be developed in AutoMOST enables implementation of a modular solution for automated vehicles considering safe behavior in different scenarios, particularly in settings structured at urban speeds (ports, urban and inter-urban areas). Hence, the AutoMOST work methodology is based on the architecture shown in Figure 5 which is depicted below.

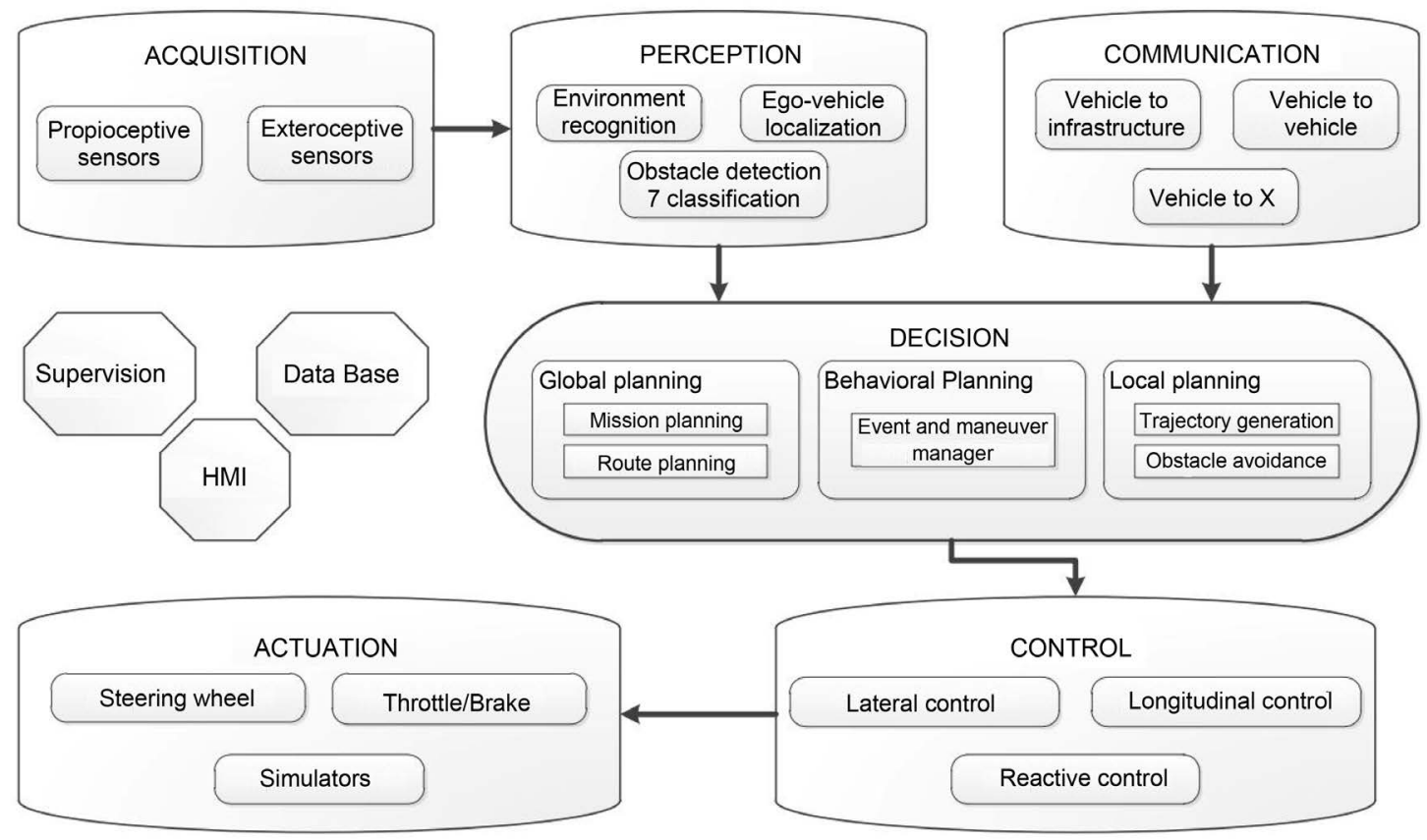

Figure 5. General abstract of automated vehicle control architecture. 
This architecture is based on the different advances developed by different international research groups and centres: California PATH, Karlsruhe Institute of Technology (KIT), the University of Parma (and Vislab), Carnegie Mellon University (CMU), Darpa Grand Challenge, Hanyang University, INRIA, etc.

Architectural modularity is a key aspect of the AutoMOST project, since it ensures the adaptability of the system to different computational environments. On another side, the greatest contribution of this architecture is reflected in the dynamic route generator, which takes the conditions of vehicle, infrastructure and obstacles into consideration in defining the route to follow and alternative routes in real time: This block represents the intelligence of the vehicle, which will allow it to negotiate different environments.

To date, most of the contributions to autonomous driving have focused on the modules for perception, communication and activity. AutoMOST focuses on the development of the modules shown in the following figure, with particular emphasis on the decision-making module, in which on-board intelligence will enable more solid control of the different situations in urban environments. The figure below shows the distribution of contributions from each partner, as per the principal on-board vehicle modules. Figure 2 and Figure 3 shows the distribution of contributions from each partner, as per the principal on-board vehicle modules.

\subsubsection{Structure of Activities}

Figure 6 describes the principal technical activities to be conducted in the $\mathrm{Au}$ toMOST project in accordance with the following activity diagram.

- Activity 1: Definition of the solutions presented in the project, in particular scientific and technological objective 1, related to implementing a control architecture for different types of automated vehicle (buses in urban settings and vehicles for infrastructure surveillance).

- Activity 2: Conduct of all tasks related to environmental recognition, mainly along three lines: positioning, obstacle detection and awareness of
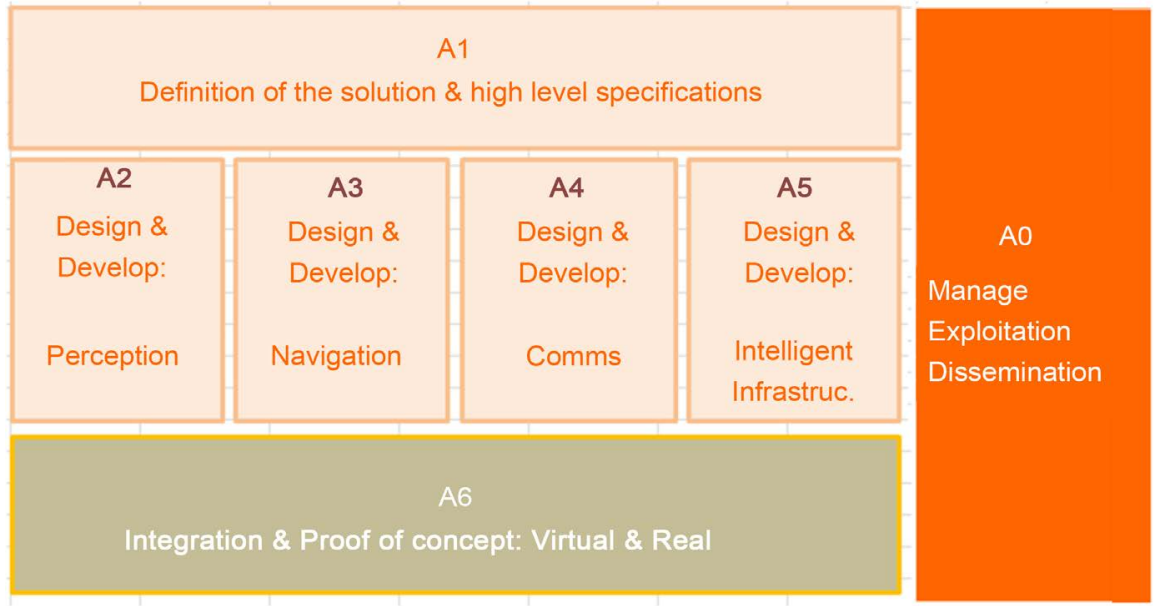

Figure 6. AutoMOST activity diagram. 
infrastructure status.

- Activity 3: Conduct of the tasks related to on-board intelligence: design of direction controllers (lateral driving) and pedal controllers (longitudinal driving), and the implementation of a global route and local path generator for the real-time definition of routes to follow, using the data from the positioning and obstacle detection system and the definition of optimum velocity profiles based on data from 3D maps, communication systems, vehicle models and propulsion system status as reference.

- Activity 4: Implementation of a communications system between the vehicles and the infrastructure (V2I) making it possible to optimize previously defined profiles and routes and warn about some emergency situations.

- Activity 5: Development of infrastructure systems with a central control center for safely operating the automated vehicle system while managing overall city traffic.

- Activity 6: Implementation and testing of the subsystems developed in activities A2-A5 in all use cases: Automated electric bus for passenger transport at the Málaga port and smart vehicles for infrastructure maintenance.

The diagram in Figure 7 shows the timeline for the technical activities projected, including the most relevant milestones.

\section{Resolution}

The AutoMOST project is resolved to develop technologies enabling vehicle automation for application to urban transport and thoroughfare status monitoring, with a view to significantly increasing efficiency, safety and sustainability. It is for this reason that a high degree of innovation may be expected as a result of

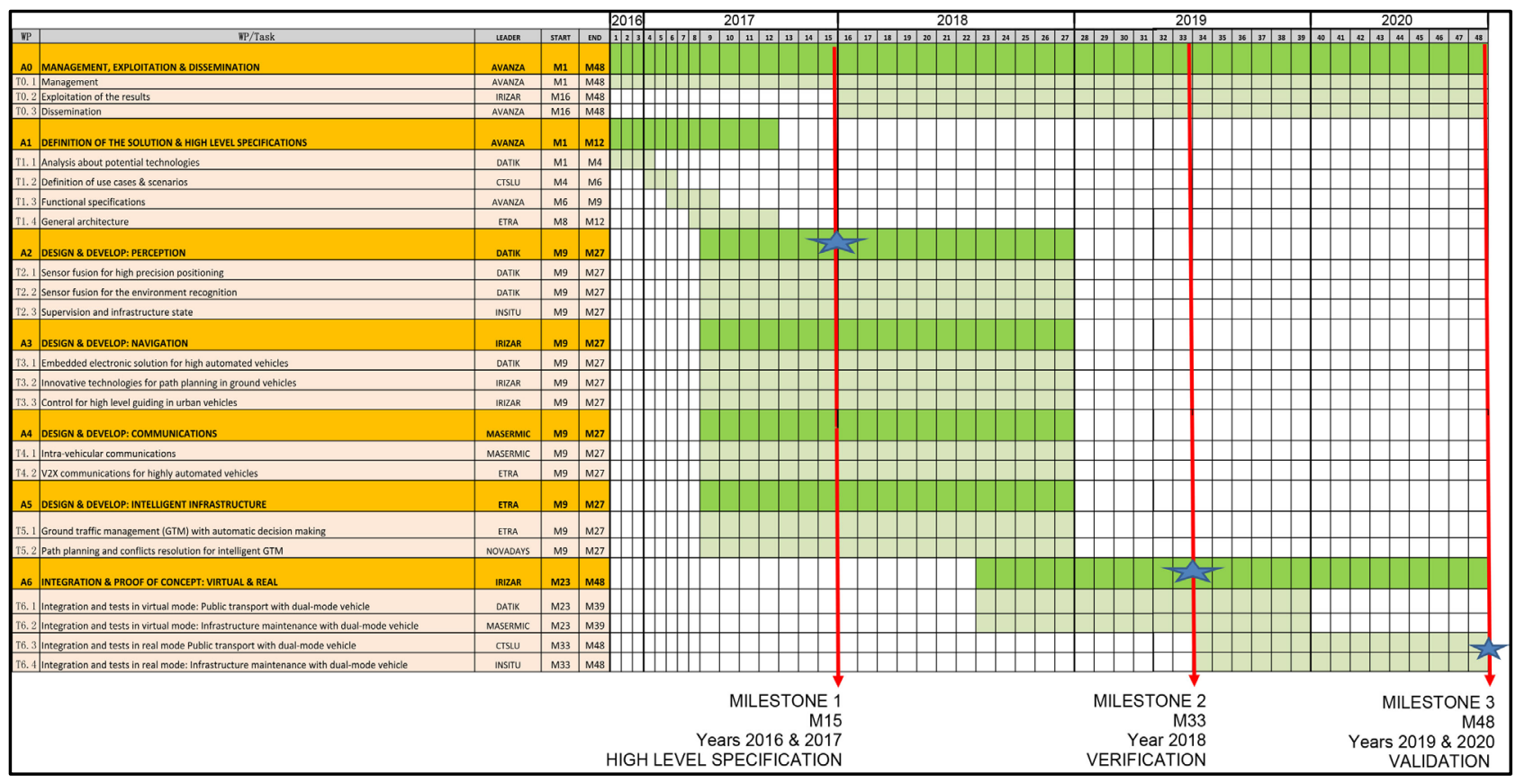

Figure 7. AutoMOST project gantt diagram. 
the project. The following are therefore outstanding among other innovations:

- Integration of the results from different disciplines to automate an electric bus for transport in the urban setting, as well as a vehicle for thoroughfare infrastructure monitoring.

- Development to implement the blocks of a modular control architecture for autonomous vehicles.

- Development to implement a multi-sensor perception solution for autonomous vehicle positioning in urban settings.

- Development of an on-board system operating in real time to generate curve parameters based on data from on-board perception systems.

- Development of a global route generator based on the data from on-board communication systems and sensors.

- Development of a dual system that will enable drivers to choose the type of activators to use in driving, as it is carried out for platoons [10] [11].

\section{Conclusions}

The challenges identified in research on both navigation and driverless vehicle control systems in dynamic urban environments are at a relatively low stage of maturity. Hence, up to now, many of the capabilities for avoiding accidents with potential obstacles and facilitating cooperative manoeuvring between automated vehicles have been limited by the scope of development projects, which usually resolve very specific scenarios under highly controlled circumstances [12], making deployment hard in complex environments, where multiple circumstances exist. This makes a decided effort for implementation in real urban settings necessary [4]-[13].

It may thus be concluded that AutoMOST focuses on the use of many of the functions already existing for automated vehicles up to the present, avoiding additional efforts with regard to systems already tested and validated. AutoMOST, however, seeks to go beyond by developing technologies that enable automated and "dual mode" driving under real traffic conditions, interacting safely with the dynamic urban environment and with expert drivers to validate interaction between different automation levels and thus allow for safe and efficient deployment.

\section{Acknowledgements}

This work is supported by the Centre for the Development of Industrial Technology (CDTI) through the CIEN (Consorcios de Investigación Empresarial Nacional) Spanish program. So, the authors would like to thank the CDTI which is a Public Business Entity, answering to the Ministry of Economy and Competitiveness in Spain, which fosters the technological development and innovation of Spanish companies. It is the entity that channels the funding and support applications for national and international R\&D\&I projects of Spanish companies.

\section{References}

[1] ERTRAC: European Road Traffic Research Advisory Council (2013) Multi-Annual 
Implementation Plan for Horizon 2020, March 2013. http://www.ertrac.org/

[2] European Commission, Roadmap to a Single European Transport Area (2011) Towards a Competitive and Resource Efficient Transport System, 2011.

[3] ERTRAC: European Road Traffic Research Advisory Council (2015) Automated Driving Roadmap, 2015. http://www.ertrac.org/

[4] Mitchell, W.J., Borroni-Bird, C.E. and Burns, L.D. (2010) Reinventing the Automobile: Personal Urban Mobility for the 21st Century. MIT Press, Cambridge, MA, 225 p.

[5] Larburu, M. and Sanchez, J. (2010) Safe Road Trains for Environment: Human Factors' Aspects in Dual Mode Transport Systems. 17 th World Congress on Intelligent Transport Systems, Busan, Korea, 25-29 October 2010.

[6] Mohanarangam, K., et al. (2014) Strategic Insight into Voice of European Consumers on Passenger Car Safety Systems, Desirability and Willingness to Pay for Active Safety and ADAS Features, Frost \& Sullivan.

[7] Natarajan, P., Jayaraman, K., Chandrasekar, P. and Ford, N. (2014) Strategic Analysis of the European and North American Market for Automated Driving Frost \& Sullivan.

[8] SAE (2014) International On-Road Automated Vehicle Standards Committee Taxonomy and Definitions for Terms Related to On-Road Motor Vehicle Automated Driving Systems SAE International.

[9] ERTRAC: European Road Traffic Research Advisory Council (2011) Working Group on Road Transport Safety and Security, European Roadmap on Safe Road Transport. http://www.ertrac.org/

[10] Solyom, S. and Coelingh, E. (2012) Performance Limitations in Vehicle Platoon Control. Proceedings of the 15th IEEE Intelligent Transportation Systems Conference, Anchorage, US, 16-19 September 2012.

https://doi.org/10.1109/ITSC.2012.6338689

[11] Robinson, T., Chan, E. and Coelingh, E. (2010) Operating Platoons on Public Motorways: An Introduction to the SARTRE Platooning Programme. 17 th World Congress on Intelligent Transport Systems, Busan, Korea, 25-29 October 2010.

[12] ADAM: Automation Development for Autonomous Mobility (2014) Iniciativa financiada por el programa INNPRONTA, 2011-2014.

[13] Parent, D. (2011) CYBERCARS: New Developments and Future Applications. Transportation Research Board 88 th Annual Meeting. 